\title{
Política urbana y hábitat popular. Nuevas dinámicas de gestión del suelo privado en Pehuajó, Argentina \\ Urban politics and popular habitat. New dynamics of private land management in Pehuajó, Argentina
}

María Angélica Ginieis

FLACSO-Argentina

Buenos Aires, Argentina

gineismariaangelica@gmail.com

\section{RESUMEN}

A fin del siglo XX, en Argentina, los procesos de reforma del Estado con la descentralización de competencias a provincias y municipios en áreas vinculadas a la salud, la educación y la vivienda, trajo consigo el problema de que la descentralización de las competencias no fue correspondida con el traslado de recursos. Hoy, se suman otros reclamos en los espacios locales relacionados con el acceso al suelo urbano y la vivienda digna, la sustentabilidad ambiental, la equidad de género, la disminución de la brecha digital entre otras. La falta de planificación de políticas integrales de hábitat para pobladores de menores ingresos, desposeídos, migrantes, empleados en servicios en las familias consolidadas o en la ciudad, profundiza la desigualdad social y la degradación ambiental en la ciudad. Este trabajo propone el análisis de las nuevas dinámicas de acceso al suelo de manera legal implementadas desde el Estado en Pehuajó, Argentina y, para ello hace foco en el estudio crítico del uso de instrumentos normativos en relación a la incorporación de la tierra privada como políticas de inclusión de la población de bajo recursos de barrios populares. La propuesta se articula como interpretación afiliada con el discurso del Programa para América Latina y el Caribe del Instituto Lincoln de Políticas del Suelo. Desde una óptica autodefinida como progresista aplica nuevos instrumentos a la gestión del suelo urbano con la función de prevenir la informalidad y tener así una ciudad más inclusiva, mejor financiada, distribuir más equitativamente los servicios públicos y las cargas, y elegir las geotecnologías más apropiadas.

Palabras claves: desigualdad urbana; gestión del suelo; inclusión; justicia social; política de hábitat

\section{ABSTRACT}

At the end of the 2oth century, in Argentina, the processes of State reform with the decentralization of competences to provinces and municipalities in areas linked to health, education, and housing, brought with it the problem that the decentralization of competences was not matched by the transfer of resources. Today, other claims are added in local spaces related to access to urban land and decent housing, environmental sustainability, gender equity, narrowing of the digital divide among others. The lack of comprehensive habitat policy planning for lower-income, deposed, migrant, service-employed people in consolidated families or in the city deepens social inequality and environmental degradation in the city. This work proposes the analysis of the new dynamics of access to land in a legal way implemented from the State in Pehuajó, Argentina, and, for this purpose, puts a critical focus on the use of normative instruments in relation to the incorporation of private land as well as policies for the inclusion of the population of low resources of popular neighborhoods. The proposal is articulated as an affiliated interpretation with the speech of the Program for Latin America and the Caribbean of the Lincoln Institute for Soil Policy. From a self-defined as progressive perspective, it applies new instruments to urban land management with the function of preventing informality and thus having a more inclusive, better-financed city, distributing public services and cargo more equitably, and choosing the most appropriate geo-technologies.

Keywords: urban inequality; land management; inclusion; social justice; habitat policy 


\section{Introducción}

A fin del siglo XX los procesos de reforma del Estado aplicados en Argentina con la descentralización de competencias a provincias y municipios en áreas vinculadas a la salud, la educación y la vivienda, trajo consigo el problema de que la descentralización de las competencias no fue correspondida con el traslado de recursos. Como analiza Basualdo, "En realidad esta transferencia de responsabilidades buscó el equilibrio fiscal de los Estados nacionales más que el fortalecimiento y el desarrollo local de las instancias locales" (2013, p. 266). Hoy, se suman otros reclamos en los espacios locales relacionados con el acceso al suelo urbano y la vivienda digna, la sustentabilidad ambiental, la equidad de género, la disminución de la brecha digital entre otras. El municipio, de un rol de proveedor de servicios cambió a acciones más proactivas con funciones ampliadas de gestión social y ambiental que Catenazzi y Reese (1998) denominan "municipalización de la crisis del Estado".

De la mano del cambio y ampliación del rol municipal devinieron nuevas problemáticas como la segregación social, conflictos por el acceso a la tierra, a la vivienda y a los servicios por las limitantes ambientales de expansión urbana territorial. La falta de planificación de políticas integrales de hábitat para pobladores de menores ingresos, desposeídos, migrantes, empleados en servicios en las familias consolidadas o en la ciudad, profundiza la desigualdad social y la degradación ambiental en la ciudad. El riesgo se fue construyendo por la desigualdad urbana y se tornó en una "ciudad injusta".

Las políticas públicas municipales desde 2007 tuvieron un importante giro en el proceso de gestión que se profundizó desde el 2013, resultando un hecho político de significativos niveles organizativos integrado por multiplicidad de agentes. Frente a ello, este trabajo propone el análisis de las nuevas dinámicas de acceso al suelo de manera legal implementadas desde el Estado en Pehuajó (Argentina) y, para ello pone foco crítico al uso de instrumentos normativos en relación a la incorporación de la tierra privada como políticas de inclusión de la población de bajo recursos de barrios populares'.

Por el modus operandis del proceso de gestión, la propuesta se articula como interpretación afiliada con el discurso del Programa para América Latina y el Caribe del Instituto Lincoln de Políticas del Suelo. Desde una óptica autodefinida como progresista aplica nuevos instrumentos a la gestión del suelo urbano con la función de prevenir la informalidad y tener así una ciudad más inclusiva, mejor financiada, distribuir más equitativamente los servicios públicos y las cargas, y elegir las geotecnologías más apropiadas.

\section{Principios y lógicas que subyacen a los procesos de producción del suelo urbano}

Referirnos al acceso al suelo articulado a las políticas de inclusión de los habitantes de Barrios Populares, trae aparejado referirse a la vivienda y en este caso de estudio a la "vivienda estatal". Para Castells, la vivienda es un producto con características específicas, un elemento esencial de la reproducción de la fuerza de trabajo y asocia el proceso de acceso a la misma con el calificativo de "penuria" porque suele resultar muy difícil la satisfacción de la necesidad que suscita. Si bien el autor realiza el análisis desde un contexto situado en la ciudad industrial francesa de los 70, las herramientas de análisis que brinda se integran adecuadamente a la realidad en estudio (Castells, 1974). Especialmente cuando hace referencia a cómo interpretar el problema de la vivienda, considera como ya se expresó que es una "penuria" y que la misma responde a una relación entre la oferta y la demanda determinada por las condiciones sociales de producción y situación en el mercado. No se trata tampoco de una situación coyuntural y de simple equilibrio entre la oferta y la demanda sino de un desfase entre las necesidades culturales, biológicas, de abrigo que es la función original de la vivienda como "valor de uso" pero también se articula con la cuestión económica por su valor de cambio. Castells argumenta que para problematizar sociológicamente la vivienda debemos centrarnos en el "análisis del proceso de producción de este bien durable, en su diversidad de calidades, de formas, de estatutos y siempre en la relación con el mercado económico y, en consecuencia, con el conjunto social en el que se inserta" (1974, p. 180).

\footnotetext{
1 Entiéndase por "Barrio Popular" a aquel con las características definidas en el capítulo XI del Decreto Reglamentario 2670 de 2015 , de la Ley Régimen de Regularización Dominial para Integración Socio Urbana (Ley 27453, 2018): Barrios populares a los barrios vulnerables integrados con un mínimo de $\mathrm{OCHO}(8)$ familias agrupadas o contiguas, en donde más de la mitad de la población no cuente con título de propiedad del suelo ni con acceso regular a al menos DOS (2) de los servicios básicos (red de agua corriente, red de energía eléctrica con medidor domiciliario y/o red cloacal).

2 Zapata se pregunta ¿qué vivienda no es social? Y agrega que denominarla "vivienda de interés social" es abonar a la estigmatización, una característica o un atributo que hace que la vivienda sea considerada como parte de un lugar inferior, inaceptable o negativo (Webconferencia
} Taller de Vivienda FLACSO, 2020). 
La producción de la vivienda resulta de la articulación de tres elementos: el terreno en el que se construye, los materiales y/o elementos de construcción y la construcción propiamente dicha. Este trabajo va a hacer centro en el acceso al terreno y específicamente en las nuevas dinámicas de gestión en el acceso al suelo mediante el uso de la recuperación de plusvalías urbanas. Es interesante explorar previamente las lógicas que subyacen a los procesos de producción del suelo mediante el uso de los instrumentos de captación de "plusvalías", preguntarnos qué autores opinan sobre ese tema, así como, explorar sobre posibles disrupciones entre elles para el tratamiento del problema.

Al respecto, Martin Smolka, integrante del Programa para América Latina y el Caribe del Instituto Lincoln de Políticas del Suelo, sobre la Recuperación de plusvalías en América Latina dice:

Por recuperación de plusvalías se entiende la movilización de parte (o, al límite, de la totalidad) de aquellos incrementos del valor de la tierra atribuible a los esfuerzos de la comunidad para convertirlos en recaudación pública por la vía fiscal (a través de impuestos, tasas, contribuciones y otras formas) o más directamente en mejoramientos in loco en beneficio de los ocupantes y la comunidad en general. Esas plusvalías resultan en general de acciones ajenas al propietario (los incrementos de valor de la tierra debido a mejoramientos realizados por los propietarios son la excepción), y más notablemente derivan de la actuación pública, sea a través de inversiones en infraestructura o de decisiones regulatorias sobre el uso del suelo urbano. A pesar de eso, estos incrementos del valor de la tierra, sin una intervención por parte del sector público para su recuperación, son apropiados en forma privada por los propietarios de la tierra beneficiada (2001, pp. 14-15).

Se trata de la "movilización de parte de los incrementos de valor" incluye no solo los instrumentos de carácter fiscal o regulatorio, sino también otros instrumentos, como la concesión, la comercialización o participación de los terrenos que permitan la absorción de plusvalías por parte del Estado. Según argumenta se trata de un incremento de valor y no de precio; es sobre un valor potencial referido al beneficio conferido al propietario del terreno, que no siempre se traduce en un aumento de precio.

Para Smolka los planificadores urbanos en Latinoamérica si bien tienen acceso a las herramientas de recuperación de plusvalías no las comprenden por completo y aun comprendiéndolas puede que no las utilicen en toda su potencialidad por falta de voluntad política. Considera que son herramientas que bien aplicadas sus ingresos pueden ser útiles:

... para regularizar ocupaciones de áreas mal o no urbanizadas, o áreas urbanizadas aún no ocupadas, representan en efecto una oportunidad para los propietarios de la tierra de imponer un premium (carga adicional) en el precio de la tierra ofrecida en el mercado informal. (2003, p. 75)

En cuanto a las negatividades de la aplicación de las herramientas señala que se observan tres tipos de casos presentes en distintas jurisdicciones y son las siguientes: a. recuperación de valor cuando no hay la intención de hacerlo o no se está consciente del hecho; b. pérdida de oportunidades para recuperar plusvalías; c. recuperación de plusvalías llevada adelante, pero con efectos perversos o resultados redistributivos negativos.

Otra mirada es la que presentan Jajamovich et al., (2018). Para dar una opinión situada decidieron tender varios hilos de análisis en el contexto de la CABA para revisitar las investigaciones vinculadas a las dinámicas del suelo urbano y el mercado residencial e interrogarse sobre el accionar tanto de grandes desarrolladores urbanos como de los pequeños y medianos a fin de reformular el discurso crítico sobre las transformaciones -y gestiones- urbanas en curso; realizaron una serie de entrevistas semi-estructuradas con informantes clave como desarrolladores inmobiliarios, gestores urbanos, expertos y académicos para entender cómo conciben la producción de la ciudad y siguiendo a Pírez (1995) indagar sobre cuáles son las lógicas políticas, económicas, de la necesidad y del conocimiento que les atraviesan. 
Otro marco de interrogantes que se analizaron:

\begin{abstract}
La cuestión de los gobiernos autodefinidos como progresistas o de izquierda y la regulación del mercado del suelo urbano; el asunto de la recuperación de plusvalías urbanas y la retención del suelo en relación a la dinámica de actores desplegadas en torno a distintos proyectos urbanos; la cuestión de la participación y su eventual institucionalización repensada respecto a las dinámicas que se despliegan en diversas iniciativas urbanas; la agenda actual de (grandes) proyectos urbanos en la ciudad de Buenos Aires (Jajamovich et al., 2018, p. 3).
\end{abstract}

El debate planteado por los/as investigadores propone que las discusiones sobre las dinámicas del suelo y el mercado inmobiliario en la ciudad de Buenos Aires debe trascender el análisis de los beneficios económicos en los asuntos urbanos, ampliar el foco más allá de los análisis técnicos y profundizar la dimensión política. Uno de los ejes críticos que subyace en relación a la captura de plusvalías es la transparencia, anticorrupción de esos usufructos, de allí plantean la necesidad de un debate de agenda ampliada que lleve al debate público, previo al uso de las herramientas, mediante prácticas participativas y democráticas prestando atención a si las herramientas colaboran o no en reducir las desigualdades sociales y urbanas en la búsqueda de ciudades más justas.

Finalmente agregar la mirada de Jaramillo (2004) que consultado en el marco de su participación en la apertura del Curso sobre mercados del suelo de la Universidad de General Sarmiento y el Lincoln Institute of Land Policy expresó que en relación al uso de la captación de plusvalía las miradas epistemológicas deberían centrase en revisar las relaciones anteriormente puestas en que los que invertían en lo inmobiliario hacían simultáneamente ganancias como capitalistas y como especuladores, esa fusión en algunas circunstancias, comienza a ser problemática. Otra observación es sobre el escepticismo de otros/as colegas técnicos e intelectuales pues considera que hay propuestas sociales que para los distintos actores se vuelven opciones reales, y eso puede ser la obra de grupos de personas, incluso de sectores que tienen sus propios intereses, pero también su propio rol. Cree que ha habido acumulación, y que buena parte de las transformaciones se dan también porque esas opciones se han diseñado, han tomado cuerpo, ha habido experiencias. Hoy se tienen justificaciones teóricas, y eso también es importante. Expresa:

...en la experiencia colombiana en el uso de instrumentos sobre todo del pago de obras públicas a partir de los incrementos en los precios de los terrenos o los inmuebles beneficiados, pero se han dado, en la última etapa, saltos interesantes, instrumentos que no solamente financian la obra pública, sino que efectivamente tratan de recuperar todas la acciones, o todos los impactos positivos en los precios del suelo, que están asociados a acciones del Estado. Acciones que muchas veces no son de inversión, sino que se trata de cambios en la legislación, en la normativa. Eso ya apunta al eslabón central de la especulación, los propietarios o los agentes que se benefician de esas mutaciones muy rápidas en los precios del suelo ligados a incorporaciones de urbanización o a cambios de normativa. Ese instrumento es muy interesante: es muy ambicioso, y sin embargo se está mostrando que es posible hacerlo. Que es compatible con nuestras legislaciones, con nuestra dinámica urbana; que no es un sueño utópico de extremistas, sino que es posible y que incluso, como tú lo señalabas, una parte de los actores, de los inversores, ve que eso no es algo que los afecte negativamente, sino que los favorece, y entonces paradójicamente hay sectores importantes de este sector de la construcción que apoyan estos procesos (S. Jaramillo en Corti, 2004, SJ: parr.8).

Las nuevas metodologías de intervención evidentemente dan cuenta de la necesidad de nuevas formas de organización y nuevas formas de investigación e interpretación. La complejidad y desigualdad urbana ha producido nuevos riesgos, conflictos y exclusiones que deben ser relevadas en el contexto de grandes intervenciones urbanísticas, pero también ha impulsado el surgimiento de nuevos paradigmas enmarcados en otras subjetividades, identidades y actores sociales que buscan concretar y ampliar nuevos derechos. 


\section{El contexto: nuevas dinámicas de urbanización}

En el Noroeste de la provincia de Buenos Aires se ubica la región de la Pampa Arenosa que se halla atravesada por la ocurrencia de períodos de inundación y de sequías, así como, de emergentes lluvias torrenciales que afectan el desarrollo armónico de la región. Desde el punto de vista geomorfológico la región se caracteriza por presentar en el área del Partido de Pehuajó un ambiente de depresión estructural que habilita la mayor retención de aguas en el sector. La ciudad de Pehuajó, construida sobre antiguas dunas presenta un relieve suavemente ondulado con espacios altos y bajos en su interior y en el norte la intersectan dos lagunas: La Salada y el Bajo Albarado.

Figura 1. Ubicación geográfica de Pehuajó. Provincia de Buenos Aires. Argentina.

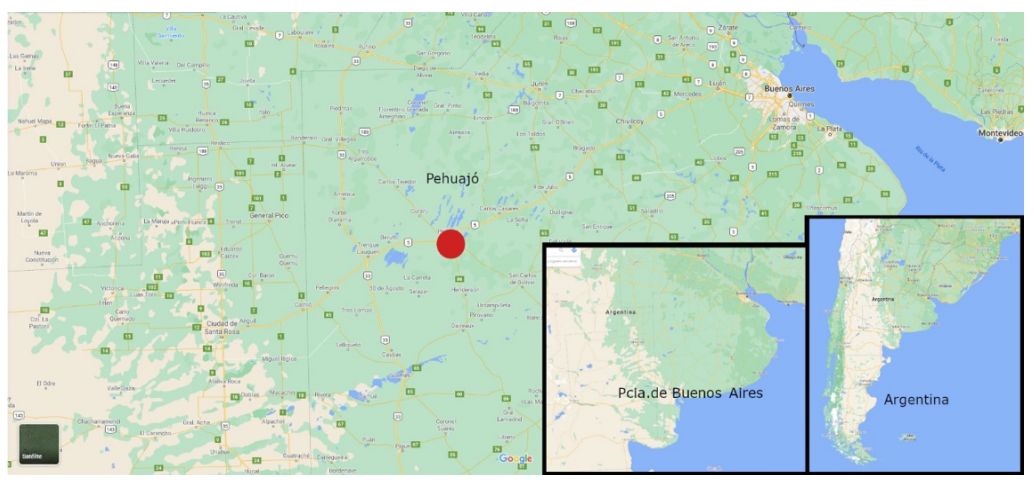

Fuente: Google Maps. Consultado 15 de diciembre de 2020.

A partir de 1973 comenzaron a darse en el Partido de Pehuajó y alrededores importantes inundaciones con un pico muy significativo en 1987. Para evitar el avance del agua sobre la ciudad de Pehuajó, se construyó un terraplén de protección que circunvala a la ciudad pero que dificulta el desagüe rápido ante lluvias torrenciales provocando sistemáticamente anegamientos en la periferia urbana. Frente a esta situación ambiental el planeamiento urbano se ha orientado a proteger la parte del centro histórico de la ciudad y no ha planteado políticas de integrales de hábitat.

La fragilidad en Pehuajó, no es solo ante lluvias, sino que se trata de una región que es escenario de eventos hidrológicos extremos (sequías e inundaciones) de distinto grado de gravedad a lo largo del tiempo. Muchos de ellos ocasionaron catástrofes mientras que otros solo alteraron las condiciones normales de algunas áreas. Hay un alto riesgo de suelos saturados y por ende de inundaciones durante el otoño hasta el verano, en años de El Niño. La última inundación importante que para el territorio bonaerense significó pérdidas económicas. Empezó a fines del 2000, ya que en noviembre los suelos se hallaban saturados, lo que significa que su capacidad de absorción y almacenaje estaban en su límite y la altura de la napa freática a muy pequeña profundidad y alcanzó el punto crítico en 2002.

Esta situación de alto riesgo hídrico recurrente hace que el suelo urbano disponible para vivienda con aptitud hídrica se transforme en un bien escaso. La demanda de "tierra vacante"3 ha ido creciendo, la Administración actual inició un proceso de relevamiento desde la Oficina Técnica Municipal de la Secretaría de Obras Públicas de la Municipalidad de Pehuajó entre los meses de Septiembre y Octubre de 2007, que arrojó el resultado que en el ejido urbano existían un total de 1907 terrenos baldíos, 250 de los cuales se encontraban en la zona identificada en el Código de Ordenamiento Territorial como RU1 (Recuperación Urbana 1) ubicados en el bajo intermedanoso. En 2013 en un nuevo relevamiento se contabilizaron 836 lotes libres entre privados y municipales. Identificada la vacancia se inició un proceso de ocupación, priorizando la urgencia de ocupación en los terrenos municipales y con los privados se aplicó una suba de hasta el 70\% del Impuesto de Barrido y Limpieza y se completó con mejoras de servicios las áreas más complejas am-

3 Tierra vacante se entiende por tierra vacante a la tierra privada no utilizada, y que se encuentra subdividida en parcelas denominadas "urbanas" según la legislación vigente (o pasibles de serlo), dentro del perímetro de la aglomeración (definido por INDEC) y que podrían ser usadas para fines residenciales, industriales, comerciales y de servicios, así como a la tierra de propiedad fiscal desafectada de sus anteriores usos. No se incluyen en esta categoría a las áreas de protección ecológica y de uso público, mientras mantengan esos usos (Clichevsky, 2006, p.2). 
bientalmente lo que originó una revalorización de los terrenos, venta y ocupación. En seis años del 70\% de los mismos ya estaban ocupados y llegando al 2014 al 93,92\%, tan solo 6,08\% de lotes estaban disponibles. Estos datos se enfrentaron igualmente a un registro para el momento de 3.200 familias con necesidad de vivienda a lo que se suman las familias de recursos medios que no están consideradas en esa estadística.

\subsection{Nuevos marcos legales de intervención}

Teniendo en cuenta que el crecimiento de la ciudad en el sentido vertical se encuentra condicionado en el Código de Planeamiento Urbano (Ord. 62/oo) por las limitaciones en la densificación de la población, debido a las deficiencias que presentan las redes de agua y cloacas, pero también debido a la morfología urbana y al género de vida de la población. La escasez de terrenos para relocalización de población en riesgo ante inundaciones, el fuerte crecimiento de la demanda de terrenos ligada a la implementación del plan PROCREAR y a la recuperación adquisitiva de los sectores medios, además de la necesidad de espacio para emprendimientos de grandes superficies, el municipio siguiendo las orientaciones del Plano Director -que si bien no ha sido actualizado sirvió de base para realizar el saneamiento de áreas señaladas de recuperación urbana- fue pergeñando estrategias de recuperación ambiental para optimizar la aplicación del instrumento de plusvalía a fin de habilitar el paso de suelo privado a público en la zona señalada como de Ampliación Urbana.

El instrumento utilizado en Pehuajó para gestionar suelo privado fue el cobro de valorización generada por el Estado como una de las estrategias para resolver el déficit de suelo urbano. La recuperación de plusvalías en este caso por parte del sector público fue una porción de los incrementos en el valor del suelo atribuibles a los "esfuerzos comunitarios" más que a las acciones de los propietarios (Smolka y Furtado, 2001a; 2001b). Para ello, según expresa la Ordenanza N 47/14 la Señora Olga Dominga Bassart de De La Peña ofreció en donación con cargo formulado a la comuna. El área ocupa una superficie de 1198 ha, de la cuales aproximadamente 148 ha se encuentran en un bajo intermedanoso y de alta vulnerabilidad ambiental que hacen inconveniente su uso residencial. Los propietarios de la chacra mencionada manifestaron su interés en acordar con el municipio la sesión de tierras correspondientes para su inclusión en el ejido urbano con la donación del $20 \%$ de las parcelas útiles para dominio municipal además del pago de un coeficiente por el incremento del valor del suelo.

La recuperación de estos incrementos puede hacerse indirectamente mediante su conversión en ingresos públicos en forma de impuestos, contribuciones, exacciones y otros mecanismos fiscales, o directamente mediante mejoras locales para beneficio de la comunidad por entero. Según los objetivos políticos de los gobiernos, dichos recursos podrían ser aplicados para mejorar la situación de acceso al suelo de los sectores sociales de menores ingresos. Pero uno de los problemas que enfrentan los gobiernos locales para la utilización de los recursos obtenidos con fines específicos, es la dificultad en términos constitucionales, de poseer cuentas especiales adonde colocar los recursos ingresados por recuperación de valorización. A menudo acaban colocándose en la cuenta general del municipio, con lo cual no es asegurado su uso a los fines previamente especificados. Cuando el cobro de valorización es realizado, el gobierno local captura parte de ese aumento de precio de la tierra, en este caso por el cambio de uso del suelo autorizado, variaciones de densidad y conversión del uso de la tierra agrícola a urbano (Clichevsky, 2006).

Este instrumento permite que las ciudades y los propietarios pueden negociar pagos en efectivo, en especie (por medio de la transferencia de parte de las tierras), o a través de la combinación de pagos en especie (tierras) y la formación de una sociedad de desarrollo urbano entre los propietarios, la ciudad y los promotores. En el caso de Pehuajó como ya se expresó se transfirió el $20 \%$ de las tierras y además a medida que el propietario al que se le habilitó el loteo va vendiendo los lotes el municipio va recuperando un porcentual de la plusvalía que logró con el cambio de uso.

Los procesos previos que acompañaron fueron, en uno de los laterales que coincide con uno de los accesos a la ciudad la construcción de acceso lateral pavimentado para el acceso al sector; en septiembre de 2011 el Ministerio de Obras Públicas de la Provincia de Buenos Aires licitó la obra de construcción de la red de agua potable 9 de julio - Pehuajó con la impulsión de pozos, estación de bombeo y anillo de refuerzo obras que permitirán mejorar la eficiencia, el caudal y calidad de la red de agua potable de la ciudad; en marzo de 2013 se firmó el contrato para la obra de electrificación de media tensión (13,2kW) Henderson-Pehuajó, con un presupuesto oficial de \$ARS 83.000.000, para un plazo de ejecución de 420 días, garantizando la calidad y eficiencia del servicio. 


\section{Cuestionamientos y análisis del proyecto de intervención de acceso al suelo}

De acuerdo a la información del municipio, los resultados de la distribución permitieron 400 lotes para el municipio y aproximadamente 1000 para el propietario del campo, estos datos no son claros pues no ofrecieron información concreta. En cuanto al tamaño del loteo varía y con ello la cantidad de lotes. La ordenanza menciona lotes de $300 \mathrm{~m}^{2}$ para el municipio, pero en la Oficina Técnica mencionaron lotes de $250 \mathrm{~m}^{2}$. Al momento de la consulta ya todos los lotes municipales están usados para la construcción de viviendas. Las viviendas debían ser las consideradas de "Interés Social” y de hecho 198 lotes fueron utilizados con el Plan Techo Digno, se especificó la inclusión de 2 Planes PROCREAR (se desconoce cantidad de lotes) y 60 lotes para viviendas que financia la provincia de Buenos Aires. En ese contexto también se incluyeron 100 lotes en un Plan de Círculo Cerrado creado por el municipio que no es para familias pobres ya que deben adjudicar con un monto inicial y luego las cuotas son de valor significativo.

Los equipos técnico-políticos municipales se valieron del antecedente de las Ordenanzas 3184/09 y 3185/09 de la Municipalidad de Trenque Lauquen que resultaron herramientas y mecanismos para la regulación del mercado de suelo, adoptando el criterio de función social de la propiedad, con la recuperación de plusvalías urbanas para invertir en desarrollo urbano. De esta manera, el Municipio comenzó a generar suelo urbano y los lotes fueron asignados al municipio para la construcción de viviendas. Los actores involucrados en el proceso, fueron los gubernamentales y los propietarios de las tierras como no gubernamentales. El alto grado de acuerdo político entre el gobierno Nacional y el representante del gobierno local fueron determinantes para el acceso a recursos financieros para la inversión urbana y la implementación de la política. La relación con el Estado provincial si bien fue de menor intensidad, permitió la articulación con el Instituto de la Vivienda (IVBA) y la Dirección de Ordenamiento Urbano. Hubo negociaciones e interacción con técnicos provinciales que contribuyeron a orientar al municipio en la subdivisión y gestión administrativa para la inscripción de los lotes. Es de reconocer que la toma de decisiones siempre estuvo centralizada en la figura del Intendente Municipal, quien se erige como la única autoridad política.

Esta estrategia ha permitido dar solución a una parte del problema de escasez de lotes pero, quedó explicada a la comunidad como una "donación". No hubo ningún tipo de debate, ni presentación ampliada, no se consultó a equipos técnicos del lugar y se desconocen las singularidades del plan. Sólo es posible consultar la normativa con la modificación de los usos. Sería interesante como plantean Jajamovich et al., 2018, una mayor transparencia de la política pública que a nivel del interior del país y en mayor medida en ciudades intermedias como esta se desconoce este tipo de herramientas de uso de las plusvalías urbanas. En acuerdo con Jaramillo (2004) sobre la sustantividad lograda por la normativa, pero es necesario publicitar y trasparentar las acciones de gobierno, consensuarlas para una el ejercicio de una ciudadanía de derecho.

El compromiso de los/as funcionarios/as políticos y en este caso mandatarios/as es cumplir con la comunidad y que el desempeño esté regulado por un diseño institucional de rendición de cuentas desde la accountability. Este es un patrón de gobernabilidad para el cuál se requiere - redefinir el rol del Estado, - redefinir las funciones que debe ejercer, - su actuación, - su modelo de gestión y profesionalización de la burocracia estratégica; se desarrollan mecanismos multidimensionales que fortalecen la participación ciudadana de manera paralela al poder que ejercen las autoridades y a la vez que consolida un mayor desarrollo político.

Probablemente entre las dificultades a superar se encuentra el aumento del valor de la tierra, la negociación de las formas de pago del coeficiente de ganancia y los avances de desarrollo urbano necesarios para la consolidación de los barrios. Al comparar los valores ex - ante y ex - post de la implantación del proyecto pueden surgir cuestiones operativas como la realización de avalúos en momentos distintos -más en el contexto de crisis - las formas y momentos de cobro, momento de las inscripciones en el registro inmobiliario, todos temas desconocidos para la comunidad y que superan el ejercicio de un mandato municipal.

Con respecto al nivel de inclusión de la población pobre urbana y la que se encuentra en espacios de vulnerabilidad ambiental no se conoce ningún plan que lo contenga por lo que es necesario visibilizar donde se cumplen la categorías asignadas en la Zona Residencial 2 (R") a la distribución de lotes para permitir la localización de Conjuntos Habitacionales de Interés Social.

Dentro de los conjuntos habitacionales que integran el espacio municipal y que según asegura la norma- 
tiva van a formar parte de los beneficiarios de los resultados de la plusvalía, siguiendo las categorías que Castells (1974) considera, la política de vivienda debe atender diferentes demandas, de acuerdo a ello se identifica que: a. Tanto la Municipalidad como la empresa privada "Raices de Ombú" que es la encargada de la venta de los lotes privados, asegura una parte del mercado de lotes para la demanda solvente; $b$. Para la población que puede ser solvente a corto plazo tanto el Estado como el privado ha establecido un cuotificación para el acceso a la vivienda en espacios donde la superficie y ubicación de los lotes resultan más económicos, aunque se desconoce cómo va a ser el acceso a servicios básicos; No se identifica una propuesta para las capas de población que sea trasformadora, inclusiva, segmentada que incluya a las mujeres jefas de familia, población LGTBI+, discapacitados/as, aquellos que integran la población insolvente con una propuesta no mercantilizada y tampoco se visibiliza una propuesta para quienes se encuentran en otros espacios de la ciudad con alto riesgo ambiental.

\section{Conclusiones}

Revisando el uso de este tipo de políticas no se registra una aplicación abarcadora en el interior de la provincia de Buenos Aires. De las analizadas no parece que estén orientadas o que hayan logrado prevenir la informalidad que es su objetivo central. Por lo que es necesario colocar los instrumentos al debate, a la contrastación y fundamentalmente planificar cómo realizar el seguimiento a largo plazo. Estos instrumentos no pueden formar parte de un currículo oculto de un municipio.

Si en realidad son políticas dirigidas a los sectores de bajos ingresos, es necesario capacitar y crear agencia a esos colectivos. Colaborar en que alcancen la capacidad de actuar independientemente y hacer sus elecciones propias de modo libre a la hora de tener que negociar para inducir a los gobiernos en el desarrollo de instrumentos con igualdad y equidad social y permitiéndoles luchar por la prioridad de quienes presentan mayores carencias.

La participación y la integración de las comunidades debe consolidarse como un proceso permanente que diluya el carácter asistencialista de las políticas, descarte el clientelismo y transforme los trabajos en redes jerárquicas en trabajos en malla de complementariedad horizontal y no de relación únicamente vertical. Es la manera de gestionar "desde abajo" hacia arriba desde problemáticas sentidas y vividas y no desde imposiciones desconfiguradas "desde arriba". La integración en el espacio barrial de funcionarios, profesionales y técnicos mejora la gestión.

Planificar cómo evaluar y seguir las políticas implementadas con transparencia permitirá entender los marcos normativos, la complejidad de composición actoral y tener feedback para replicar las buenas prácticas.

\section{REFERENCIAS}

Basualdo, J. L. (2013). Planes urbanos, la llegada al territorio de las estrategias del municipio. En, D. A. Erba (Org.), Definición de políticas de suelo urbano en América Latina: teoría y práctica, (pp. 263-272). Lincoln Institute of Land Policy.

Castells, M. (1974). La cuestión urbana. Siglo XXI

Clichevsky, N. (2007). La tierra vacante “revisitada”. Elementos explicativos y potencialidades de utilización. Cuaderno Urbano, 6, 195-220. http://dx.doi.org/10.30972/crn.661023

Clichevsky, N. (2013). Mercado de suelo y medio ambiente, una relación conflictiva. En, D. A, Erba (Org). Definición de políticas de suelo urbano en América Latina: teoría y práctica, (pp. 243-254). Lincoln Institute of Land Policy.

Clichevsky, N. (2006). Previniendo la informalidad urbana en América Latina y el Caribe. CEPAL. Serie Medio Ambiente y Desarrollo. https://cutt.ly/8h31Vkr

Corti, M. (junio de 2004). Entrevista con Samuel Jaramillo “Ahora existen mecanismos para corregir las distorsiones del mercado”. Café de las ciudades. https://cafedelasciudades.com.ar/economia_20.htm

Jajamovich, G., Spirtu Barros, N., \& Gentili, R. (2018). Produciendo Buenos Aires. Procesos urbanos e interrogantes rumbo a otra agenda urbana. Laboratorio de Políticas Públicas. https://cutt.ly/oh31NCK

Pírez, P. (2019). Una aproximación sociológica al derecho a la ciudad. Derecho y Ciencias Sociales, (21), 6-22. https://doi. org/10.24215/18522971e6-22

Smolka, M. y Amborsku, D. (2003). Recuperación de plusvalías para el desarrollo urbano: una comparación inter-americana. EURE, 29(88), 55-77. http://dx.doi.org/10.4067/So250-71612003008800003 
Smolka, M., y Furtado, F. (2001). Ensayo introductorio: recuperación de plusvalías en Latinoamérica: ¿bravura o bravata? En M. Smolka y F. Furtado. (eds.) Recuperación de Plusvalías en América Latina. Alternativas para el desarrollo urbano. Pontificia Universidad Católica de Chile y Lincoln Institute of Land Policy. https://cutt.ly/ghzopie

\section{AUTORA}

María Angélica Ginieis. Magíster en Género, Sociedad y Políticas; Especialización en Economía y Pobreza por PRIGEPP-FLACSO (Argentina). Tutora docente en el Diplomado en Género y Políticas Públicas y en la Maestría en Género, Sociedad y Políticas de FLACSO Argentina.

Conflicto de intereses

La autora informa que no existe conflicto de interés posible.

Financiamiento

No existió asistencia financiera de partes externas al presente artículo.

Agradecimientos

N/A. 